\title{
El Habario, un yacimiento musteriense al aire libre en los Picos de Europa cántabros
}

\author{
Elena Carrión Santafé * y Javier Baena Preysler **
}

\section{RESUMEN ABSTRACT}

En el presente trabajo se presentan los resultados del análisis industrial del yacimiento de El Habario (Pendes,

Cantabria), primer yacimiento paleolítico cántabro al aire libre excavado en un ambiente de alta montaña. La explotación sobre cantos de cuarcita implica la captación directa de los recursos líticos de altura, muy localizados en el entorno. Se describen las características industriales del conjunto, sus peculiaridades técnicas (con una cadena operativa especifica, muy homogénea y bien representada) y la funcionalidad supuesta para el mismo (taller). Tdo ello sugiere la existencia de estrategias complejas de aprovechamiento del medio. Aunque

por el momento no ha sido posible obtener dataciones absolutas, dada las características de la muestra, el
The present paper outlines the results of the industry analysis of El Habario (Pendes, Cantabria), first open palaeolithic open air site, placed at a high altitude, studied in this region. The explotation of quarzite pebbles implies the direct captation of lithic resources in a mountainous area, a particular procedure in this case. The industry characteristics are described, its technical peculiarities (with a specific operative secuence, very homogeneous and well represented), as well as the assumed funtionality of the site (worksite). All this implies the presence of a complex purposefulness in the captation. Allthough up to now it has been impossible the absolute dating, given the characteristics of the sample, the internal analysis of the industry permits us to assign the findings to the Middle Paleolithic period. In this line,

* Departamento de Prehistoria y Arqueología de la UAM.

** Profesor Titular del Departamento de Prehistoria y Arqueología de la UAM. 
análisis interno de la industria permite inscribir el conjunto en momentos del Paleolítico Medio. Así mismo se insiste en la riqueza arqueológica de la comarca de La Liébana, que cuenta hasta el momento con una importante representación en yacimientos paleolíticos y ofrecerá en los próximos años, sin duda, resultados muy prometedores. we enfatize the archaeological richness of La Liébana area, wich atpresent has a very important representation of paleolithic sites. We hope, therefor that in the comming years it will yeald very promising results.

\section{EL MUSTERIENSE AL AIRE LIBRE EN CANTABRIA}

Aunque los yacimientos musterienses con mejores secuencias y óptimo estado de conservación son aquéllos situados en las numerosas cuevas y abrigos la región, no debemos olvidar la existencia de un buen número de yacimientos musterienses al aire libre. Este grupo de testimonios van siendo poco a poco recogidos en la bibliografía peninsular y extranjera, a pesar de los problemas de interpretación que muchos de ellos presentan. Tanto en las síntesis actuales sobre el Musteriense cántabro, (Cabrera, 1984b, 1988; Cabrera y Bernaldo de Quirós, 1992) como en los trabajos generales sobre el Paleolítico Medio peninsular (por ejemplo, Vega Toscano, 1983) no suele prestarse suficiente atención a estos yacimientos, mencionándose tan sólo el conjunto de los alrededores de Altamira (estudiado de antiguo: Breuil y Obermaier, 1935, González Echegaray, 1958; revisado por R. Montes, quien descarta su atribución Musteriense: Montes, 1993) y el conjunto industrial y faunístico de Unquera, que se conoce tan sólo por vagas referencias a la trinchera del ferrocarril (Obermaier. 1925). Butzer seguiría afirmando, años después, que «(...) unfortunately, no openair mousterian sites have been documented in Cantabria" (Butzer, 1981: 179). La mayoría de los mismos aparecen recogidos en la Carta Arqueológica de Cantabria (Muñoz et al., 1987, 1988).

Muchos de estos yacimientos aparecen estudiados en trabajos más recientes (Carrión Santafé, 1998; dentro del Proyecto de Investigación Ecologia y Subsistencia de las Primeras Poblaciones Neandertales en el centro de la Región Cantábrica, financiado por la Diputación Regional de Cantabria), y han sido objeto de estudios más o menos parciales sobre aspectos concretos (Muñoz, 1982, 1988; CAEAP, 1987; San Miguel et al., 1984b; Gaem, 1993; Muñoz et al., 1991; Muñoz y Malpelo, 1992; Muñoz, 1991-1992; CAEAP/GEIS (Inédito). Existen además trabajos concretos sobre los yacimientos de Panes II (Montes y Muñoz, 1992a), la Verde A 
(Montes y Muñoz, 1992b) y El Habario (Castanedo et al., 1993; Carrión Santafé et al., 1997) únicos tres yacimientos al aire libre que han sido objeto, dentro de la Comunidad Autónoma de Cantabria (exceptuando Panes II, perteneciente al Principado de Asturias), de recogidas sistemáticas o excavaciones rigurosas.

Cierto es que gran parte de estas estaciones al aire libre presentan serios problemas de interpretación industrial y cronológica. La mayoría de estos yacimientos, salvo el caso de El Habario (en un ambiente de alta montaña, en la Comarca de La Liébana) se encuentran jalonando la línea de costa en función del aprovechamiento de sílex costero, (Fig. 1 y 2) apareciendo la industria en una pobre sucesión estratigráfica. Aunque en muchos de estos yacimientos la atribución cultural es discutible, por haber sido objeto de sucesivas visitas en momentos prehistóricos y protohistóricos, las características internas de los conjuntos (núcleos discoides musterienses, algunos tipos clásicos) permiten, a falta de otros datos, atribuirlos al Paleolítico Medio. La funcionalidad de la mayor parte de estos yacimientos, que han sido calificados en algún caso de «talleres» (Morlote y Montes, 1992), debe a nuestro juicio matizarse para hablar, mejor de "Centros de Aprovisionamiento", dado que la captación de materia prima es la principal actividad en ellos desarrollada.

Así, los conjuntos presentan gran abundancia de cantos brutos (objetos en muchos casos de tanteo y abandono, con explotación sumaria) junto a núcleos agotados y abundantes productos brutos de lascado, con probable retirada de matrices. Esta funcionalidad supuesta complementa lo observado en las cuevas de la región, donde, a la escasez de núcleos, se une la aparición en los conjuntos de materiales de probable procedencia "exótica» (es el caso del sílex de El Castillo; Cabrera, 1988), para los que se ha sugerido una procedencia litoral.

Los núcleos de estas colecciones al aire libre, aunque algunas veces se encuadran en tipos característicos, (por ejemplo, un núcleo Levallois en el yacimiento de Universidad), son en ocasiones discoides y en la mayoría de los casos "amorfos" o "globulares", producto de una pérdida de morfología que permita su adscripción a tipos como consecuencia de la intensa explotación de la que han sido objeto. Las características internas de los conjuntos (en las que, como decimos, priman los productos brutos de lascado, y, sobre todo, restos de talla y desechos) impide un estudio tecnológico de las series, y sólo destaca como caracteristica particular, en el caso de las escasas lascas presentes en ellos, la relativa abundancia de talones suprimidos (ya señalada como particular en el Musteriense cántabro, con abundante retalla de talón; González Echegaray, 1958). Puede re- 


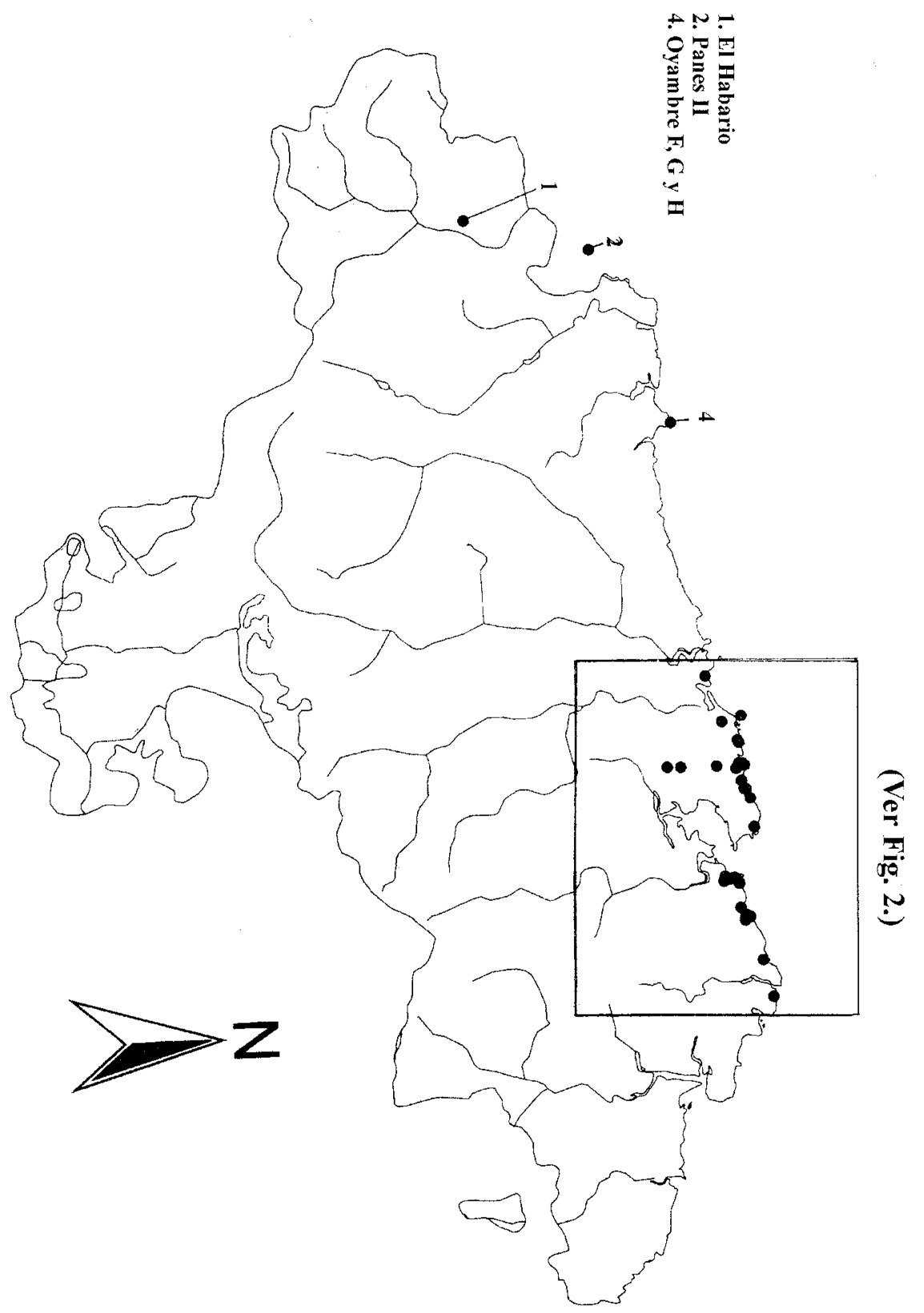

Fig. 1. Yacimientos musterienses al aire libre en Cantabria: El Habario. 


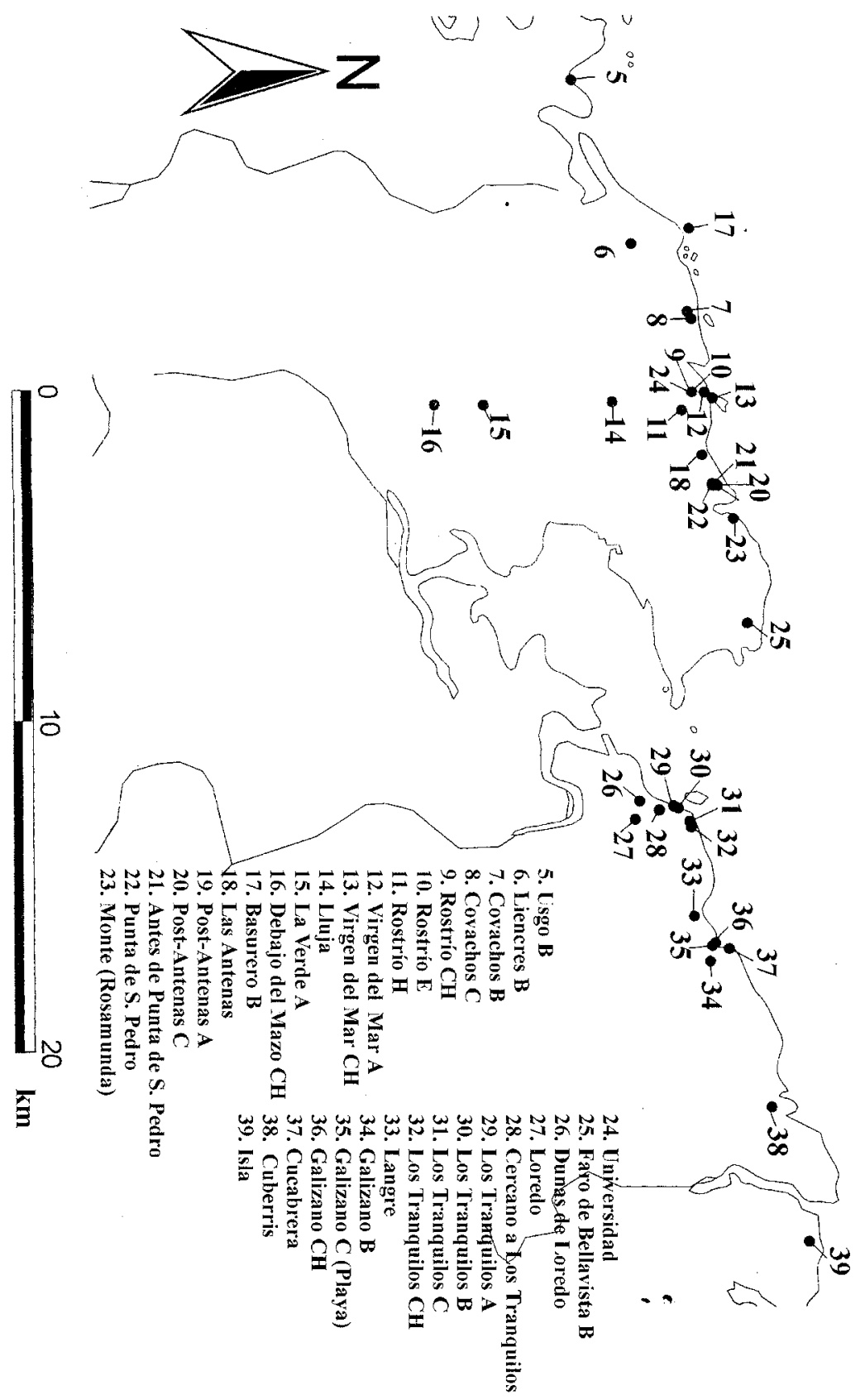

Fig. 2. Localización de los yacimientos de atribución musteriense al aire libre en Cantabria. 
conocerse en algunos casos un trabajo centrípeto, sin preparación periférica de la superficie de golpeo. Se trataría en general de un acercamiento «prueba» a la materia prima, para luego, eventualmente, comenzar la explotación hasta el agotamiento casi total del nódulo.

En el conjunto de estos yacimientos el utillaje es escaso, atípico y en la mayoría de los casos, de dudosa atribución. Aparecen en algún caso hendedores, la mayoría de tipo 0 (en algún caso, con tipos más complejos, como por ejemplo en Panes II; Montes y Muñoz, 1992a), en materiales de grano grueso (cuarcita, arenisca, ofita). Entre el utillaje destacan las raederas, los denticulados, los cantos trabajados, los cuchillos de dorso natural y las escotaduras, apareciendo en la mayoría de los casos el retoque como dudoso o marginal. No se descarta, en algún caso, la mezcla de productos de diferente cronología en los conjuntos (siendo especialmente abundantes en la zona los talleres calcolíticos; Muñoz et al., 1987).

Caso especial to constituye el yacimiento de El Habario (Pendes, Cantabria), en el que ha sido reconocida una cadena técnica muy bien definida y homogénea, con representación de todas las categorías productivas. Aunque preliminarmente fue atribuido al Musteriense Antiguo debido a las características atípicas de muchas de sus piezas unidas a criterios climáticos (a falta de posibilidades de datación absoluta), la comparación aún en proceso de análisis con cadenas operativas semejantes en yacimientos próximos en cueva (Cueva del Esquilleu, con once niveles de ocupación hasta el momento) podría adelantar más la cronología sugerida. Junto con otros yacimientos (Oyambre F.G y H y Panes II) la mayor abundancia de la fase Producción entre las categorías líticas de El Habario permite incluirlo en la categoría de «taller», a diferencia de la mayor parte de los yacimientos al aire libre estudiados, donde la cadena operativa aparece representada en sus fases más elementales.

\section{PECULIARIDAD REGIONAL DEL YACIMIENTO DE EL HABARIO}

El yacimiento de El Habario es el primer yacimiento musteriense cántabro excavado hasta el momento en un contexto de alta montaña. Ello, junto con la circunstancia de tratarse de un yacimiento al aire libre, lo hace especialmente atractivo para su estudio, desarrollado a partir de las excavaciones emprendidas en 1996 por un equipo dirigido por el Dr. Baena Preysler. La escasa alteración que presentan las piezas, incluidas en una breve secuencia estratigráfica, permite en principio garantizar la unidad de génesis del conjunto, confirmada además por la gran coherencia interna (a nivel tecnológico) del material. 
El emplazamiento del yacimiento es por sí solo un elemento de reclamo científico sobre el mismo. Hasta el momento, y salvo la Cueva de la Mora (González Echegaray, 1957a), no se habían estudiado en la comarca yacimientos con esta cronología. Esto nos lleva a plantearnos en qué medida los sistemas de asentamientos tradicionalmente aceptados para la región no se han visto condicionados por la naturaleza de las prospecciones realizadas hasta el momento, lógicamante más intensas en los alrededores de los núcleos urbanos, en la línea de costa (zonas de explotación humana intensiva) y en los cursos fluviales, aún hoy ejes económicos de la comunidad. Así, hasta el momento las zonas montañosas no han sido objeto de localizaciones musterienses de importancia, aglutinándose los hallazgos, (tanto al aire libre como en cuevas) en las proximidades de la capital y en el pasillo costero, sin adentrarse apenas en las modestas alturas de las Sierras Litorales.

Quizás por este motivo, la comarca de La Liébana ha estado especialmente abandonada por la investigacion paleolítica. El equipo cántabro CAEAP exploró intensivamente la región (Muñoz et al., 1985), descubriendo un buen número de yacimientos incluidos en la Carta Arqueológica (Muñoz et al., 1987). Actualmente el problema se va subsanando con las últimas actuaciones arqueológicas desarrolladas en la comarca, y que han ido poniendo de manifiesto una muy interesante ocupación correspondiente al Paleolítico Medio. Es el caso de la Cueva de Fuentepara, El Habario, y, sobre todo, la Cueva del Esquilleu, interesante yacimiento que se revela, en sus preliminares once niveles de ocupación, como clave para la caracterización del Musteriense en el occidente de la Comunidad (Baena, et al., e.p.). También figuran en la Carta Arqueológica regional algunos yacimientos como la Cueva de la Mora, descubierta ya de antiguo (González Echegaray, 1957b), el Covacho de la Hermida o la Cueva del Arteu (yacimiento musteriense en inminente peligro de desaparición).

El Habario se encuentra alejado $20 \mathrm{~km}$. de la costa hacia el interior (en línea recta), y unos $30 \mathrm{~km}$. sin consideramos el recorrido real siguiendo el curso del río Deva, que se constituye como el principal eje de comunicación de esta montañosa región. Se encuentra situado a 536 metros de altura, apareciendo en una suave ladera desde donde se domina el valle del río Deva y la confluencia de otros valles menores subordinados a aquél.

El nivel de las nieves perpetuas supuesto para la glaciacion Wurmiense (1350-1400 m. sobre el nivel marino; González Echegaray, 1957) y los fenómenos de glaciarismo, muy abundante en la zona, ponen límites a la 
ocupación humana, circunscribiendo la actividad del yacimiento a momentos interglaciares o interestadiales. Ello permitió en su día (Carrión Santafé, 1998) sugerir una probable cronología arcaica al yacimiento que tratamos, que carece por el momento de posibilidades de datación absoluta y que sólo ha podido ser asignado mediante un estudio interno del mismo. Sin embargo, la comparación de las cadenas operativas presentes en el mismo y algunos niveles de cronologías más recientes en la cercana Cueva del Esquilleu, cuestiona la hipótesis anterior y aproxima culturalmente ambos yacimientos.

Las posibilidades de aprovisionamiento de materia prima se ven limitadas en gran medida por el dominio geológico en el que se inscribe el yacimiento. Hacia el oeste del mismo, enormes crestas calizas marcan el comienzo de la unidad geológica de los Picos de Europa, caracterizada por la presencia de estructuras paleozoicas reactivadas (abundancia de cuarcitas y pizarras), y que se distingue del dominio que conforma el grueso de los materiales de la comunidad cántabra (materiales secundarios y terciarios plegados sobre el zócalo hundido). El yacimiento se encuadra en el Dominio de Liébana, amplia cubeta deprimida (de predominio sedimentario), rodeada de moentañas y cruzada por diversas serranías entre las que discurren los afluentes del Deva.

Ello provoca que el entorno del yacimiento proporcione, básicamente, materiales del Carbonífero, sobre todo areniscas, lutitas, conglomerados y calizas (IGME. 1: 50.000, Hoja n. ${ }^{\circ} 56$ ). Algunos de estos conglomerados, han sido localizado en las proximidades inmediatas de El Habario, y han servido claramente como fuente de aprovisionamiento para la talla, realizada en su totalidad a partir de cantos rodados de cuarcita. Los cantos, de tamaño variable, se desprenden de la roca de forma natural. La excepcionalidad en la región de tales conglomerados sugiere que el hombre paleolítico conocía la oferta del medio, y que, puesto que el propio río arrastra materiales de semejante calidad a mucha menos altura (com. pers. I. Manzano) las estrategias de aprovisionamiento lítico son más complejas de lo generalmente inferido para estos momentos, o tal vez subsidiarias de otras actividades económicas.

La estatigrafía del yacimiento es bastante sencilla, apareciendo los materiales embutidos en una capa de limo amarillento producto de la degradación de las pizarras subyacentes, características de las zonas deprimidas de la comarca por oposición a la caliza de las alturas. El rodamiento presente en el conjunto puede explicarse por desplazamientos de los materiales por solifluxión (com. pers. M. Frochoso). Este tipo de fenómenos son frecuentes en suelos situados en laderas cargadas de humedad, que 
se deslizan por pendientes moderadas. No descartamos, dada la ausencia casi total de la fracción pequeña de la industria, una selección por gravedad de los materiales.

\section{ESTUDIO DE LA INDUSTRIA}

El Habario permitió diseñar un modelo de cadena operativa en el que se engranan la práctica totalidad de las piezas encontradas. La coherencia del proceso de captación, reducción y talla, permite hablar de un proceso estandarizado, en el que los sistemas de explotación del núcleo muestran una clara uniformidad. Ello nos permite aventurar para el conjunto una cronología avanzada dentro del Paleolítico Antiguo (Musteriense). Si en un principio se habló de un cierto arcaismo en la producción (referida básicamente a la tosquedad y escasa estandarización de su utillaje; Carrión Santafé, 1998), la incorporación a su definición de criterios referidos a la propia organización de la producción (sistemas de reducción conceptualmente cercanos a lo Levallois) obliga a revisar las afirmaciones anteriores.

La colección es breve. Se compone de 517 piezas, quedando su clasificación como sigue:

\begin{tabular}{lcc}
\hline & $N^{\circ}$ Piezas & Porcentaje \\
\hline Núcleos para lascas & 62 & 11.9 \\
NÚCLEOS & 62 & 11.9 \\
& & \\
Descorticado Primario & 27 & 5.2 \\
Descorticado Secundario & 81 & 15.6 \\
Lascas simples & 120 & 23.2 \\
Lascas Levallois & 13 & 2.5 \\
Láminas & 7 & 1.3 \\
PRODUCTOS LASCADO & 248 & 47.8 \\
& & \\
Útiles & 59 & 11.4 \\
Lascas Retocadas & 95 & 18.3 \\
MATERIAL RETOCADO & 154 & 29.7 \\
& & \\
Debrises de talla & 17 & 3.2 \\
Debrises de retoque & 2 & 3.5 \\
DEBRISES & 19 & 6.5 \\
RESTOS DE TALLA & 34 & \\
\hline
\end{tabular}


La proporción de materias primas empleadas muestra un claro dominio de la cuarcita $(95.3 \%)$, seguida de la cuarcita/arenisca $(2.9 \%)$, de la arenisca $(0.7 \%)$, y, en cantidades residuales, por el sílex, el cuarzo o la caliza. Se trata de una cuarcita de grano fino (cuarcita armoricana), procedente de los cantos del conglomerado, que suele fracturarse espontáneamente siguiendo diaclasas de alteración interna, con un patrón característico, que produce tabletas de planta ovalada. Tales formas han sido aprovechadas en algunos casos directamente como matrices (sin que se haya obervado una selección clara de formatos específicos).

El silex aparece en la colección de forma puntual: los afloramientos locales, escasos y de difícil acceso, ofrecen aceptables calidades pero de escasa aptitud para la talla, debido a la abundancia de fisuras internas (Arias Cabal, 1987); los restos de este material en El Habario son escasos y de pequeño tamaño. Además el sílex aparece en la comarca en puntos muy limitados y de difícil acceso, que han sido localizados cartográficamente a unos $2.5 \mathrm{~km}$. desde el yacimiento.

\section{Núcleos}

Sorprende la homogeneidad del proceso de reducción. El sistema de explotación comienza con una captación específica de los conglomerados de cuarcita cercanos. Sobre lascas de descorticado o, preferentemente, sobre tabletas producto de la alteración o fractura de los cantos, se inicia una estrategia de explotacion centrípeta, poco intensiva, sobre la tableta o la lasca de descorticado elegida como matriz.

Tras una preparación del hemisferio cortical de la misma, centrado en la zona del talón, se procede a la explotación de la superficie de lascado de forma centrípeta, con especial atención sobre el engrosamiento bulbar de la matriz. En la mayoría de los casos no se han agotado las posibilidades de explotación sin pérdida de formato, por lo que suele quedar visible en los núcleos parte de la superficie de lascado de la matriz original.

Ello produce numerosas lascas Kombewa, en nuestro caso escasamente estandarizadas, y que son el resultado de explotar los planos de lascado de las matrices (lascas). Hasta qué punto este sistema de explotación debe clasificarse como técnica diferenciada de explotación (Santonja, 1984-85) o como el resultado de la adaptación de los esquemas productivos a los soportes empleados (lascas), es a nuestro juicio un problema que debería ser discutido con más profundidad. 
La diferenciación conceptual de ambos hemisferios, en la que uno de ellos es destinado básicamente a la preparación de la superficie de golpeo, convirtiéndose el otro en plano de explotación productiva preferente, acerca el esquema de trabajo a las reducciones jerarquizadas definidas como Levallois (Boëda, 1988a, 1993). La insistencia de la explotación en la zona próxima al talón de la matriz (en ocasiones preparado con especial atención) podría significar la asunción de ciertos criterios cercanos al concepto de predeterminación /preferencialidad característico de la modalidad lineal de esta técnica de talla. Por la propia configuración de la matriz, la relación angular entre ambos hemisferios es en muchos casos Subparalela/Secante, sin que haya sido necesaria una adecuación de las convexidades laterales de la superficie de lascado ya que el soporte de partida las presenta. Sin embargo los ángulos de lascado y las morfologías finales de los núcleos quedan condicionados por la propia configuración formal de las matrices.

En algunos casos la cadena operativa se continúa hasta que las bases negativas quedan convertidas en discoides, que sería por tanto, en $\mathrm{El}$ Habario, una fase final del proceso de reducción. La jerarquización de las superficies, así como la relación angular entre las mismas (quizás también la funcionalidad asignada a cada una de ellos) podría haberse visto modificada en sucesivas fases de tabajo.

\section{Productos de lascado}

Aunque las lascas de descorticado son relativamente abundantes (81 de 248), predominan las lascas simples (108); 27 corresponden a procesos de descorticado secundario. Los talones son lisos en un $64.4 \%$, sin que sean raros los facetados (18.8\%) y los diedros (13.0\%).

Estas proporciones son coherentes con los núcleos observados en la colección. Destaca la escasez de productos de lascado Levallois estricto, lo que está en consonancia con el patrón de explotación reconocido en el conjunto, y con las características generales descritas para el Musteriense cántabro (Cabrera, 1984b, 1988; Cabrera y Bernaldo de Quirós, 1992). A efectos orientativos, citaremos que el ILTec es 3.9, siendo el ILTipológico de 21.3. La industria es No Facetada, aunque sus índices de facetaje amplio y estricto son relativamente altos (33.8 y 19.8, respectivamente).

En las lascas predomina claramente el esquema de anverso con negativos de dirección paralelo-transversal. Ello está en consonancia con una explotación radial de dirección centrípeta, apoyando el esquema observado 
en los núcleos del yacimiento e imprimiento en el conjunto un fuerte aire de homogeneidad.

A pesar de que el proceso de excavación fue riguroso (criba sistemática) faltan lasquitas en el conjunto.Quizás los procesos de alteración arriba mencionados (en cualquier caso, de limitada intensidad) habrían producido cierta selección en el arrastre y la desaparición de la fracción pequeña de material. Las reproducciones experimentales han confirmado la abundancia que de este tipo de productos se genera en el proceso de talla.

\section{Material Retocado}

Aunque no han sido incluidas en este grupo las piezas con retoque muy marginal, sin continudad o con rotura de pátina, el material retocado plantea grandes problemas de clasificación. La presencia de denticulados podría estar sobrevalorada por la intervencion de agentes postdeposicionales (Castanedo et al., 1993). Dominan por tanto en la colección los denticulados y las raederas, con presencia de algunos perforados atípicos, escotaduras y puntas. En general los tipos coinciden con lo observable en los yacimientos al aire libre cantabro, incluyendo la presencia en El Habario de un hendedor tipo O. El porcentaje de piezas Levallois, tanto técnico como tipológico, es escaso. Queremos hacer constar además la dificultad de reconocimiento de retoque útil, dada la presencia de gran cantidad de melladuras de uso o producto de procesos mecánicos, en nuestra opinión no antrópicos.

De las 159 piezas que conforman el conjunto de material retocado, tan sólo 59 puede encuadrarse en tipologías clásicas estrictas (Bordes, 1961). Este problema se ve acusado por la abundancia de retoques denticulantes, poco regulares, que muchas de las piezas presentan, y que en principio permitió su inclusión (no sin ciertas reservas) en la facies de Musteriense de Denticulados (Castanedo et al., 1993).

\section{Proceso de trabajo}

La gran coherencia técnica del conjunto permitió definir con bastante precisión el proceso técnico desarrollado, representado en la (Fig. 3). Existe una clara uniformidad en la concepción del núcleo como unidad de explotación, dentro de un esquema técnico planificado y definible que implica la existencia de modelos previos. Este sistema de explotación, fuertemente 


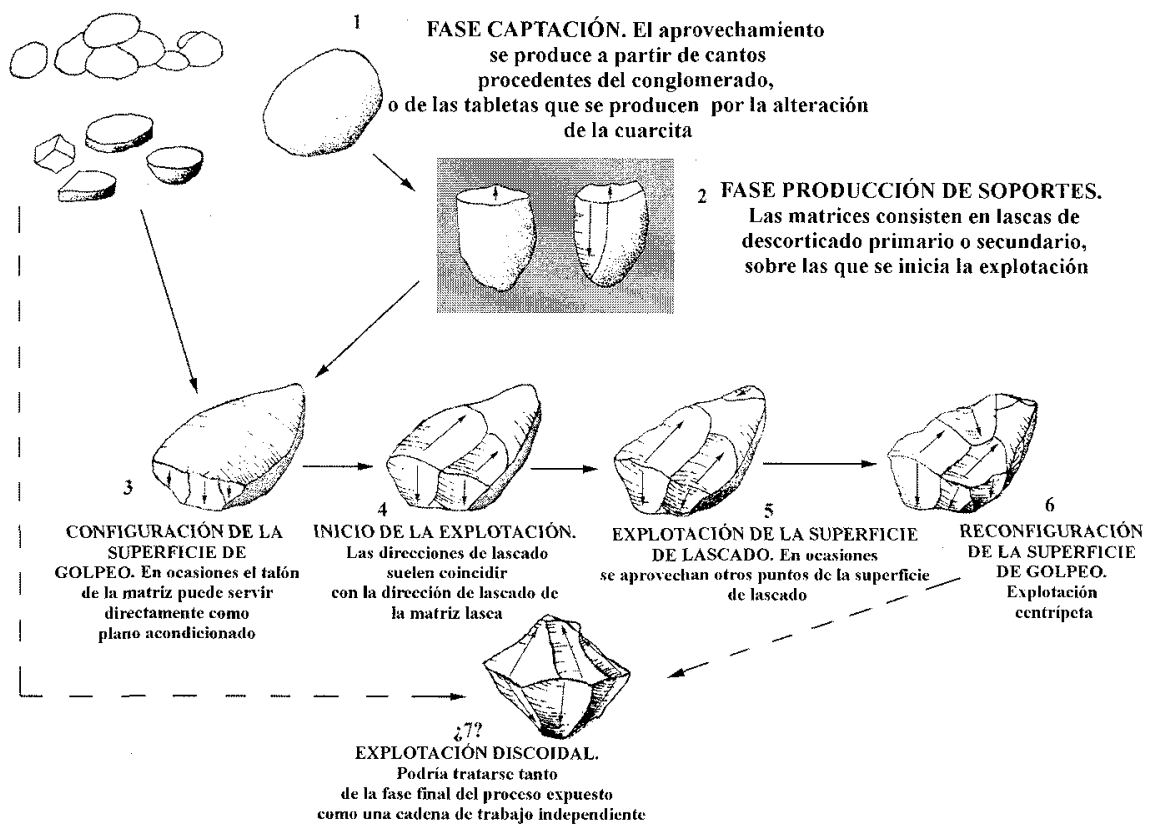

Fig. 3. Esquema de trabajo en el yacimiento de El Habario. Los núcleos de la colección reflejan el abandono de la explotación en fases sucesivas.

musteriense en su concepción, permite inducir una cierta cronología general al conjunto, a pesar de que los núcleos resultantes estén dotados de una gran personalidad. La reproducción experimental de este modelo de explotación permitió comprobar la eficacia del mismo y la contrastación positiva de los productos experimentales con el material arqueológico, con el que guardaba fuertes similitudes.

Aunque pensamos que el tema debe ser objeto de una más profunda discusión, no queremos dejar pasar la oportunidad de apuntar las dificultades de definición que, en función del modelo de explotación que venimos definiendo, hemos encontrado a la hora de caracterizar los núcleos Kombewa. Muchos de los productos de lascado presentan superficies de lascado en sus anversos, circunstancia ligada a la elección como matrices de lascas de descorticado. Ello nos ha planteado no sólo numerosos problemas de definición, sino también discusiones conceptuales a la hora de concebir el núcleo como unidad tecnológica cerrada sin posibilidad de introducción de elementos de variación individual. 
Los núcleos Kombewa pueden definirse como «... aquellos núcleos sobre lasca cuyo anverso coincide con la cara bulbar de aquélla" (Santonja, 1984-85: 28. Tixier presenta una definición semejante: « (...) le débitage de l'eclat dont la face inférieure servira de surface de débitage et l'enlèvement de l'eclat Kombewa, peut se placer (...) la préparation d'un plan de frappe»; Tixier, 1980: 55). Dada la matriz elegida en El Habario, los primeros golpes habrían producido lascas cuyo anverso mostraría parte del reverso de la misma. Posteriomente, las características Kombewa del núcleo serán enmascaradas por aristamientos, fruto de las sucesivos grados de trabajo. Los ángulos de golpeo se van modificando, quedando en algunos casos, en los núcleos con explotación intensa, una relación entre hemisferios definible como Secante/ Secante.

Encontramos por tanto que la definición de un núcleo Kombewa podría relacionarse más con el estadio de abandono de la explotación sobre una matriz característica que con criterios técnicos o culturales preestablecidos. De alguna forma, la secuencia del yacimiento de El Habario se ajusta conceptualmente a la secuencia progresiva:

1. Kombewa (matriz lasca; primer paso de la secuencia de reducción)

2. Explotación centrípeta con jerarquización funcional entre hemisferios (preparación/explotación, guardándose entre ellos en algunos casos relación angular Subparalela/ Secante). No hay acondicionamiento de convexidades (característico de esquemas Levallois), quizás por la propia morfología del soporte lasca.

3. Discoide (pérdida progresiva del formato previo, hasta el agotamiento del núcleo).

En todos los casos se ha detectado la presencia de técnica de percución directa con percutor duro.

Esta dificultad en la clasificación de modelos de reducción del Paleolítico Medio, que parte de la concepción del núcleo como un objeto dinámico sujeto a constante transformación conceptual y formal, ha sido discutida ya por algunos autores (Moncel, 1998). De hecho, la caracterización de la talla discoide por oposición a la Levallois y las posibilidades de reconocimiento de una $u$ otra modadlidad a partir de núcleos abandonados es actualmente objeto de profunda reflexión (Jaubert, 1993; Locht et al., 1995).

En varios niveles de la cercana Cueva del Esquilleu se han detectado núcleos semejantes técnicamente a los descritos (niveles III, IV, V VI). Se trata igualmente de lascas de cuarcita o caliza/limolita (u otras rocas de grano fino, no siempre identificadas), sobre las que se ha emprendido 
un trabajo centrado en la zona del bulbo de la matriz. El trabajo en el hemisferio inferior, que coincide con el talón de la lasca soporte, condiciona la relación angular entre ambas superficies, aproximándolas, si seguimos este criterios, a modelos Levallois (Subparalelo/Secante). A ello se une la clara existencia de jerarquización entre las superficies de trabajo (Com. pers. V. Requejo).

Aunque se carece hasta el momento de dataciones absolutas para el nivel en cueva al que aludimos, su situación estratigráfica (por encima de un nivel Charentiense de tipo Quina muy claro) nos aproxima culturalmente el conjunto hacia momentos finales del Paleolítico Medio. Por tanto El Habario, cuyos núcleos guardan tanta semejanza, podría ponerse en paralelo con estas cronologías tardías (Fig. 4, 5 y 6 ).

\section{FUNCIONALIDAD DEL YACIMIENTO EN EL CONTEXTO DEL MUSTERIENSE CÁNTABRO}

a) La proporción de núcleos presentes en el yacimiento en relación con el material bruto de lascado y con las piezas retocadas permite asimilar el conjunto a la categoría de taller, tal como se sugirió en un estudio previo realizado sobre materiales de superficie (Castanedo et al., 1993). La proporción ente núcleos y lascas queda de 6.4 a 1 (excluyendo los desechos y las lasquitas) y de 7.0 a 1 (excluyendo sólo las lasquitas). La proporción de lascas por núcleo en los modelos experimentales desarrollados es sensiblemente mayor.

Si El Habario funcionó como taller efectivo, habrían sido retiradas del mismo, lógicamente, matrices brutas para su retoque o uso directo posterior, que en función de las reproducciones efectuadas, habrían sido aproximadamente 5 lascas por cada una abandonada.

El porcentaje de núcleos en el yacimiento (12\%) destaca sobre lo observado en otros yacimientos cántabros en cueva (en los niveles musterienses de Morín, no se supera el 1\% de núcleos: González Echegaray y Freeman, 1970, 1973; en El Pendo el máximo está en 3.8\%: González Echegaray, 1980), que funcionan claramente como lugar central de hábitat o consumo. Para la Cueva del Esquilleu, en proceso de excavación (Baena et al., 1999), los núcleos son proporcionalmente muy escasos. En este ejemplo encontramos una clara diferenciación funcional entre lugar de captación (presumiblemente, el lecho del inmediato río Deva) y explotación inicial, frente a las categorias de la fase consumo presentes en la cueva. 

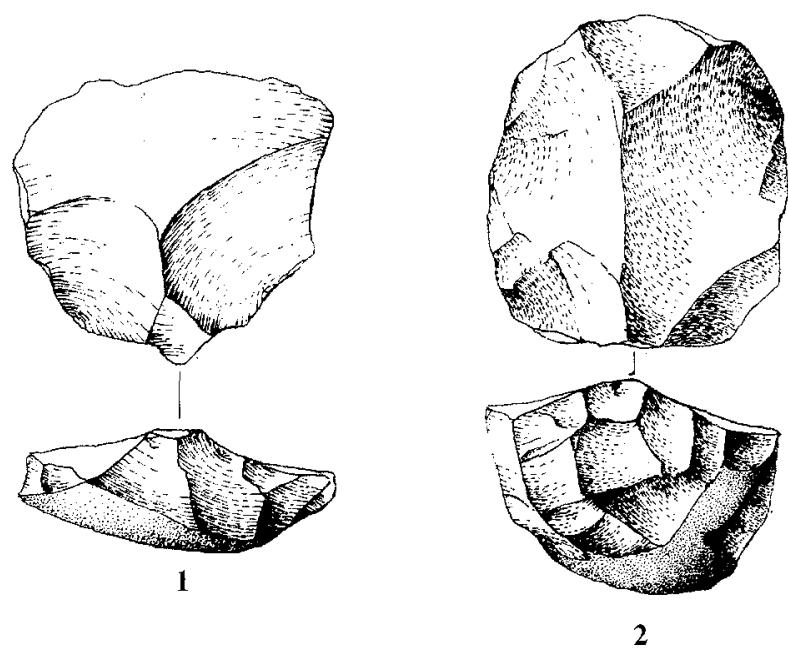

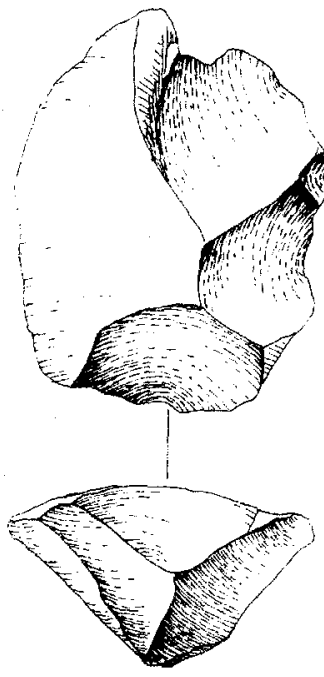

3

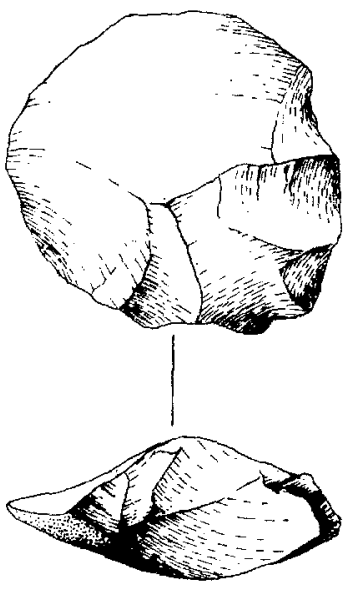

4

Fig. 4. Núcleos de El Habario. El proceso de trabajo es muy homogéneo: sobre una lasca de descorticado se produce la explotación de la superficie de lascado de la matriz, con preparación periférica del hemisferio inferior. 

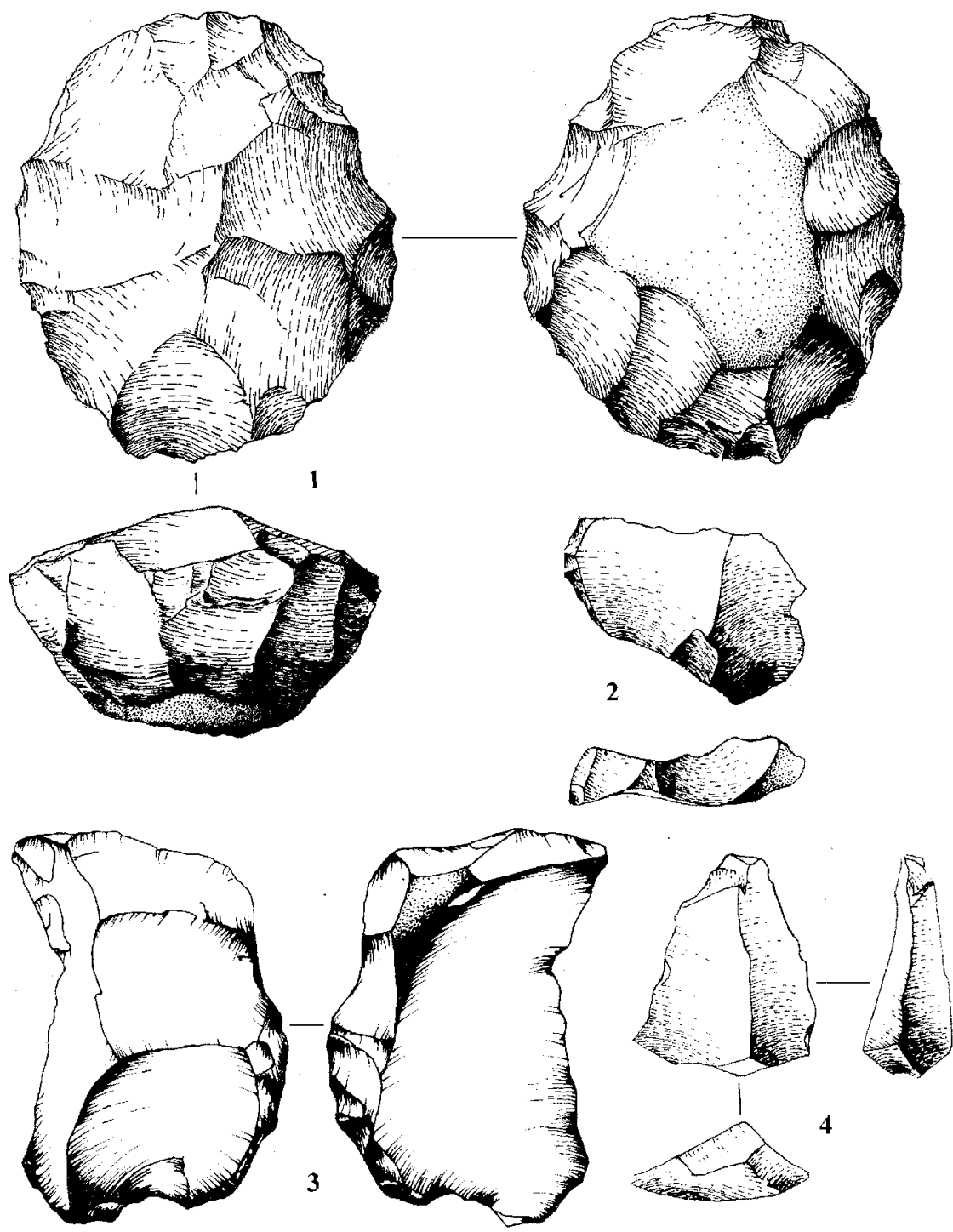

Fig. 5. 1. Núcleo Levallois del nivel superior (revuelto). 2. Lasca Levallois. 3. Lasca Levallois desbordada. 4. Punta Levallois. 

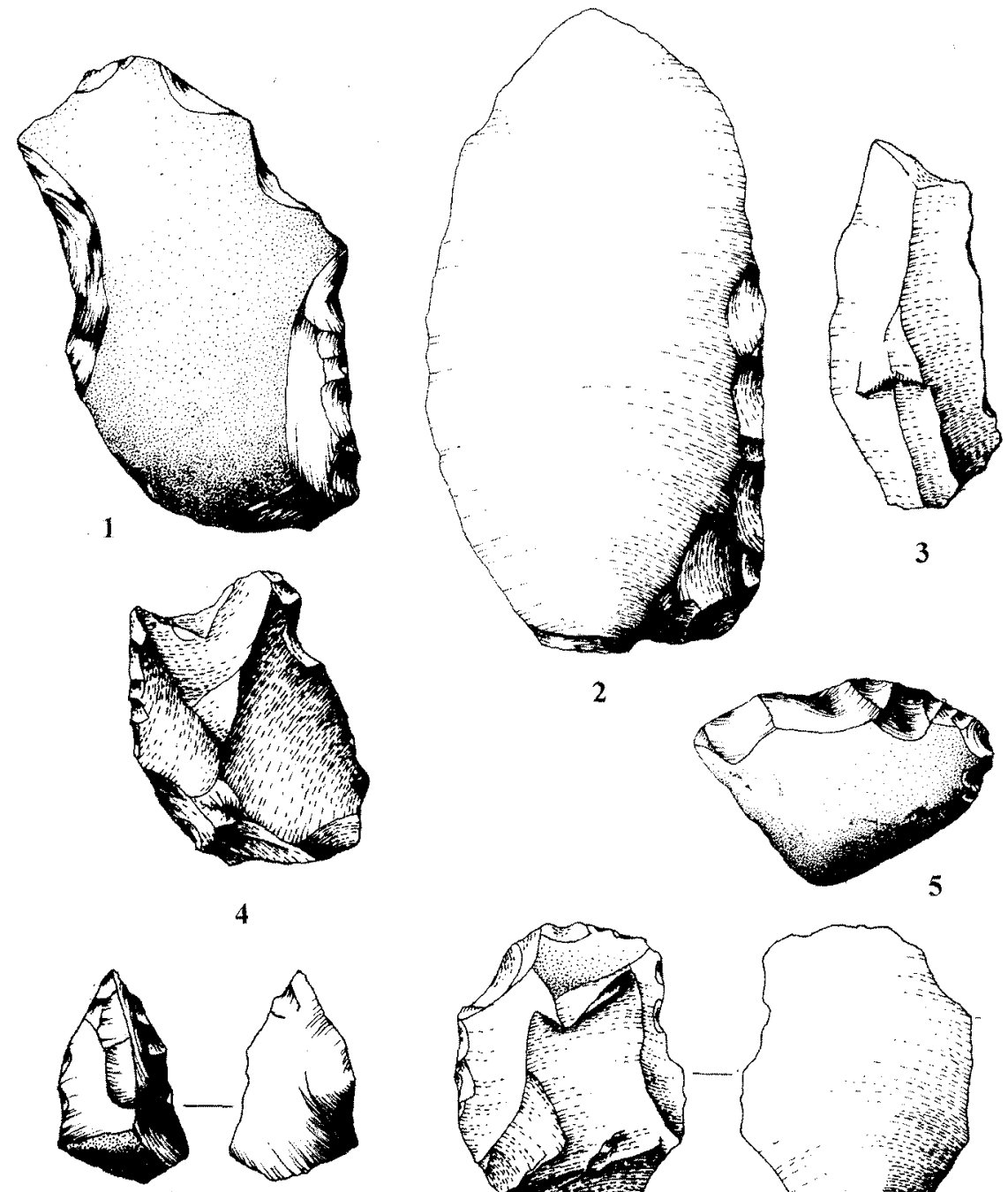

6

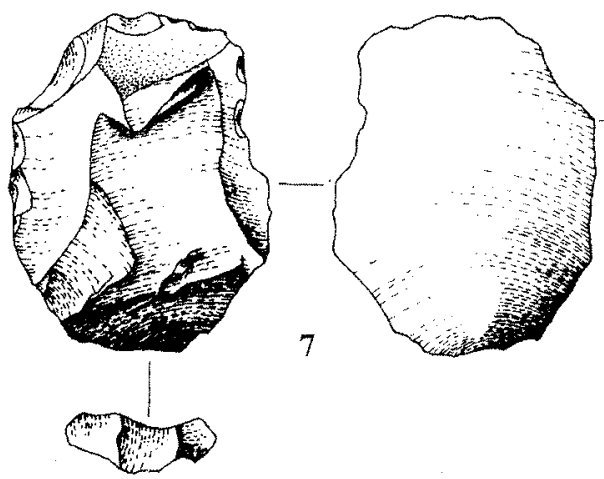

Fig. 6. Material lítico de El Habario. Las piezas son atipicas y con abundancia de pseudoretoques. 1. Raedera doble recto-cóncava. 2. Raedera sobre cara plana. 3. Pieza de orientación paralela. 4. Perforador atípico. 5. Denticulado. 6. Punta.

7. Lasca pseudoretocada. 
Distinguimos la categoría de taller de la de centros de aprovisionamiento, en la que puede englobarse, como hemos apuntado anteriormente, la mayoría de los yacimientos al aire libre situados en la Cantabria litoral. En ellos encontraríamos espacios en los que la fase de la cadena operativa representada es básicamente la fase de captación, tanteo y desbastado sumario de los cantos de sílex costeros. Otro tipo de prductos, propios de fases de la producción más avanzadas (lascas, núcleos, elementos retocados), son, coherentemente, muy escasos.

b) Buscaríamos entonces una complementariedad tecnológica y funcional a nivel de categorías líticas con las cuevas habitadas de la región. Efectivamente, la fase Consumo, escasamente representada en los yacimientos al aire libre, aumenta su representación, junto a matrices brutas de lascado. En función de un modelo de partida, quizás excesivamente simplista pero sin duda sugerente, podemos esbozar una primera explotación de recursos en los yacimientos al aire libre (categorías nucleares con escasos grados de trabajo, restos de talla, fragmentos de materia prima inclasificable), con retirada de productos brutos de lascado, complementada con los centros de hábitat (cuevas), donde encontramos porcentajes mucho más elevados de lascas, utillaje, y, en su caso, de núcleos (escasos) objeto de explotación intensiva.

Todo ello implica la existencia de centros nucleares a nivel funcional y espacial durante el musteriense cantabro, donde, a diferencia del Achelense local (Montes, 1993), cuya estrategia ha podido ser definida como de inmediatez, hay un conocimiento del territorio (zonas de captación-transformación en complementariedad funcional con las zonas de consumo) y se aplica una lógica al uso ecológico del mismo. Entendemos la diferenciación funcional musteriense en escalas espaciales variables, sin perjuicio de que las distancias entre lugares de captación y de consumo durante el Paleolítico Medio estén muy próximas (por ejemplo en la propia Cueva del Esquilleu).

Sólo nos queda llamar la atención sobre el original aprovechamiento de los conglomerados de El Habario (de localización muy precisa en la comarca). Puede explicarse de dos formas:

A) Proximidad al centro de habitat, de la que este punto de aprovisionamiento sería una especie de estación «satélite». Aunque se han realizado prospecciones y sondeos en el entorno, especialmente en las proximidades (Cueva de Cabañes, con escasas piezas de cuarcita), hasta el momento no han sido localizadas ocupaciones intensas en el entorno cercano. No olvidemos que el río Deva arrastra numerosos cantos de materias primas variables (entre ellas cuarcita) que podrían haber sido aprovechados 
con facilidad. La explotacion de los recursos de altura sugiere por tanto la posibilidad de presencia cercana de centros de agrupación más o menos estables.

B) El aprovechamiento lítico, en el caso de El Habario, podría haber sido subsidiario de algún otro tipo de actividad de captación (cinegética, por ejemplo, preferentemente aplicada sobre especies de roquedo)., por lo que el hábitat asociado a estos grupos podría localizarse en distancias medias. En las estrategias económicas de estas comunidades deberían engranarse, además del aprovechamiento de materia prima litica, otras necesidades (proteína animal, recursos vegetales, cercanía a ríos o surgencias de agua, competencia con otros grupos humanos o animales, condiciones climaticas específicas, etc.) que presentan sin embargo gran dificultad de interpretación a partir del registro arqueológico.

\section{BIBLIOGRAFÍA}

BAENA, J., et al. (e. p.): «Avance de los Trabajos Realizados en el Yacimiento Paleolítico de la Cueva del Esquilleu (Castrocillorigo, Cantabria)". Actas del 3. ${ }^{\circ}$ Congreso de Arqueología Peninsular. Vila Real (Portugal), 21-27 de Septiembre de 1999.

BOËDA, E. (1988a): «Le concept Levallois et evaluation de son champ d'application». OTTE, M. (Ed.): L Homme de Néandertal. Vol. 4. La Technique. Lieja. págs. 13-26.

BOÉDA, E. (1988b): «Le concept laminaire: rupture et filiation avec le concept levallois». OTTE, M. (Ed.): L'Homme de Néandertal. Vol. 8. La Mutation. Lieja, págs. 41-59.

BOËDA, E. (1993): «Le débitage discoïde et le débitage Levallois récurrent centripète». Bulletin de la Societé Préhistorique Française. Tomo $90, n^{\circ}$ 6, págs. 392-404.

Breull, H. y Obermaief, H. (1935): The Cave of Altamira ar Santillana del Mar, Spain. Madrid.

BUTZER, K.W. (1981): «Cave sediments, Upper Pleistocene stratigraphy and Mousterian Facies in Cantabrian Spain: The Acheulian/Mousterian Question". Journal of Archaeological Science, vol. 8 (2). págs. 133-183.

CABReRA VALDÉs, V. (1984b): «El Musteriense en Cantabria». Boletín Cántabro de Espeleología, n. ${ }^{\circ}$ 4. Las Culturas Prehistóricas en las Cuevas de Cantabria. Santander, págs. 28-45.

CABRERA VALDÉS, V. (1988): «Aspects of the Middle Paleolithic in Cantabrian Spain». OTTE, M. (Ed.): L Homme de Néandertal. Vol. 8. La Mutation. Lieja. págs. 27-37.

CABRERA VAldÉs, V. y Bernaldo DE QuiRós, F. (1992): "Approaches to the Middle Paleolithic in Northern Spain". Dibble, H.L; Mel.lars, P. (Eds.): The Middle Paleolithic. Adaptation, Behaviour and Variability. Univ. Pensilvania. págs. 97-112.

Carrión Santafé, E., Baena, J., Conde, C. (1997): «Avance de los procesos de excavación realizados en el yacimiento de El Habario (Pendes, Cantabria): Procesos Técnicos". CUPAUAM, n. 22.

Carrión SAntAfé, E. (1998): El yacimiento de El Habario (Pendes, Cantabria) y su relación con las industrias al aire libre en el centro de la Región Cantábrica. Memoria de Licenciatura. Universidad Autónoma de Madrid (Inédito).

CAEAP (1987): "Yacimientos prehistóricos. Informe arqueológico de Oyambre-San Vicente". Oyambre, espacio natural. Santander, págs. 89-93, 154.

CAEAP/GEIS: Carta Arqueológica de Ribamontán al Mar. (ínédito).

CASTANEdo, 1. et al. (1993): «El yacimiento al aire libre de El Habario (Castro-Cillorigo, Cantabria)". Nivel Cero, n. ${ }^{\circ}$ 3. Santander. págs. 153-175.

GAEM, SC (1993): Informe del seguimiento arqueológico de urgencia del tramo de la Autovía del Cantábrico BEZANA-LAS LLAMAS (Santander). Agroman-MOPTMA (Inédito). 
GonZÁlez ECHEgARAY, J. (1957a): «La Cueva de la Mora, un yacimiento paleolítico en la región de los Picos de Europa". Altamira, n. ${ }^{\circ}, 2$ y 3 . Santander págs. 3-26.

GonzÁlez ECHEGARAY, J. (1957b): «Las glaciaciones de los Picos de Europa y sus relaciones con los yacimientos de la costa cantábrica».AGuIRre, E. (Ed.): Actas del $V$ Congeso Internacional del INQUA Madrid-Barcelona, 1957. Tomo I. págs. 243-248.

GONZÁlez ECHEgaRAY, J. (1958): «El Paleolítico Inferior en los alrededores de Altamira». Altamira., n. ${ }^{\circ}$ 1-3. Santander. págs. 349-369.

Gonzalez Echegaray, J.; Freeman, L.G. (1970): Cueva Morín. Excavaciones 1966-1968. Patronato de las Cuevas Prehistóricas de Santander.

González Echegaray, J. y Freeman, LG (1973): Cueva Morín. Excavaciones 1969. Patronato de las Cuevas Prehistóricas de Santander.

González Echegaray, J. (1980): El yacimiento de la Cueva del Pendo. Excavaciones 19531957. Biblioteca Prehistórica Hispana, n. ${ }^{\circ}$ 17. Madrid.

IGME (1984-1994): Mapa Geológico de España (1:50.000). Hojas 56 y 81.

JAUBERT, J. (1993): «Le gisement paléolithique moyen de Mauran (Haute-Garonne): Technoéconomie des industries lithiques". Bulletin de la Socie te Préhistorique Française, Tomo 90, n. ${ }^{\circ}$. págs. 238-335.

LOCHT, JL, et al. (1995): «Le gisement paléolithique moyen de Beauvais (Oise)». Bulletin de la Societé Préhistorique Française, Tomo 92, n. ${ }^{\circ}$. págs. 213-226.

MONCEL, MH (1998): «Les niveaux moustériens de la grotte de Saint-Marcel (Ardéche). Fouilles René Gilles. Reconoissance des niveaux á débitage discoïde dans la vallée du Rhône". Bulletin de la Societé Préhistorique Francaise. Tomo 95, n. ${ }^{\circ}$ 2. págs. 141-170.

MONTES BARQUín, R. (1993): Los complejos industriales del Paleolítico Inferior en el centro de la región cantábrica. Trabajo de Investigación del Tercer Ciclo. Universidad de Cantabria (Inédito).

MONTES, R. y MUÑOz, E. (1992a): «Un nuevo yacimiento de superficie en Asturias: Panes II (Peñamellera Baja, Asturias)". Boletín del Real Instituto de Estudios Asturianos. Sección Ciencias, $\mathrm{n}^{\circ}$ 42. Oviedo.

MONTES, R. y MUÑOz, E. (1992b): «Informe arqueológico del solar sito en el barrio de La Verde (Herrera de Herrera), propiedad de la empresa 'Canteras Transmiera'»-Consejería de Cultura y Deportes del Gobierno de Cantabria.

MORLOTE, JM y MONTES, R. (1992): «Las estaciones del Paleolítico Antiguo desde Rostrío hasta Cabo Mayor». Nivel Cero, ${ }^{\circ}{ }^{\circ}$ 1. Santander, págs. 9-22.

MuÑOz, E. et al., (1982): “Carta Arqueológica del Valle de Piélagos». Altamira, n. ${ }^{\circ}$ XLIII. Santander. págs. 245-307.

MUNNOZ, E. et al. (1985): "Yacimientos arqueológicos en el Valle del Deva». Boletín Cántabro de Espeleología, n. ${ }^{\circ}$. 6. Santander. págs. 67-74.

Muñoz, E. y MAlPelo, B. (1992): Carta Arqueológica de Camargo. Ed. Ayuntamiento de Camargo. Santander.

MuÑoz, E. et al., (1987): Carta Arqueológica de Cantabria. Ed. Tantín. Santander.

MuÑoz, E. et al. (1988): Carta Arqueológica de Santander. Ed. Tantín. Santander.

Muñoz, E. et al., (1991): "Carta Arqueológica de los municipios de Argoños, Arnuero, Escalante, Meruelo y Noja». Arquenas, n. ${ }^{\circ}$ 1. Santander. págs. 2-59.

Obermaler, H. (1925): El Hombre Fósil. Madrid, 1985.

San Miguel, C. et al. (1984): "El Achelense en Cantabria». Boletín Cántabro de Espeleología, n. ${ }^{\circ}$ 4. Las Culturas Prehistóricas en las cuevas cántabras. Santander. págs. 18-27.

SANTONJA, M. (1984-85): «Los núcleos de lascas en las industrias paleolíticas de la Meseta española". Zephyrus, $n .^{\circ}$ XXXVII-XXXVIII. Salamanca. págs. 7-13.

Vega TosCANo, L.G. (1983): “Los problemas del Paleolítico Medio en España». Homenaje al Profesor Martín Almagro Basch, 1. Madrid, págs. 115-130. 